\title{
Potencialidade da Educação Permanente na prevenção da infecção pelo Covid-19 em profissionais de saúde: relato de experiência
}

\author{
Potential of Permanent Education in the prevention of Covid-19 infection in health \\ professionals: experience report
}
Potencial de la Educación Permanente en la prevención de la infección por Covid-19 en profesionales de la salud: informe de experiencia

Renata Lopes do Nascimento Santos ${ }^{1,2 *}$, Laysa Fernanda de Almeida Sousa Lima ${ }^{1}$, Rubiane Gouveia de Souza e Silva ${ }^{1,2}$, Sandra Regina Silva de Moura ${ }^{1,2}$.

\section{RESUMO}

Objetivo: Relatar a experiência de treinamentos realizados sobre as medidas preventivas adotadas durante a pandemia do COVID-19, visando o fortalecimento e empoderamento por parte dos profissionais. Relato de experiência: Trata-se de um relato de experiência, apresentado em ordem cronológica e de natureza descritiva. Vivenciada em uma instituição de saúde, referência para atendimento de pacientes com COVID19. Durante a execução dos treinamentos, observou-se o fortalecimento da Educação Permanente dentro da Instituição. A procura pelo conhecimento sobre as medidas de prevenção e as discussões sobre a melhor forma de cuidar do paciente, passou a ser interesse de todos os profissionais que estavam direta e indiretamente ligados ao cuidado com o paciente. Neste cenário foram treinados 2.152 profissionais, em 215 grupos. Considerações finais: Acreditar na importância do trabalho realizado pela educação permanente é perceber que é possível educar, de forma humanizada, que todos os profissionais são integrantes do processo de aprendizagem, e que é essencial a compreensão das necessidades e angústias vivenciadas como parte da construção do novo saber dentro do ambiente de trabalho.

Palavra-chave: Profissionais de saúde, Infecções por coronavírus, Educação permanente.

\begin{abstract}
Objective: To report the experience of training conducted on the preventive measures adopted during a COVID-19 pandemic, classic the strengthening and empowerment of professionals. Experience report: This is an experience report, presented in chronological order and of a descriptive nature. Experienced in a health institution, a reference for the care of patients with COVID-19. During the execution of the training, it was observed the strengthening of Permanent Education within the Institution. The search for knowledge about preventive measures and as a consequence about the best way to care for the patient, became the interest of all professionals who were directly and indirectly linked to the care of the patient. In this scenario, 2,152 professionals were trained in 215 groups. Final considerations: Believing in the importance of the work carried out by permanent education is perceived that it is possible to educate, in a humanized way, that all professionals are members of the learning process, and that it is essential to understanding of the needs and anxieties experienced as part of the construction of the new saber within the work environment.
\end{abstract}

Keywords: Health personnel, Coronavirus infections, Education continuing.

\section{RESUMEN}

Objetivo: Reportar la experiencia de capacitación realizada sobre las medidas preventivas adoptadas durante una pandemia de COVID-19, clásico del fortalecimiento y empoderamiento de los profesionales. Informe de experiencia: Es un informe de experiencia, presentado en orden cronológico y de carácter descriptivo. Experimentado en una institución de salud, referente para la atención de pacientes con COVID-19. Durante la ejecución de la capacitación, se observó el fortalecimiento de la Educación Permanente dentro de la

\footnotetext{
${ }^{1}$ Instituto de Medicina Integral Prof. Fernando Figueira (IMIP), Recife - PE. *E-mail: educa@imip.org.br

${ }^{2}$ Faculdade Pernambucana de Saúde (FPS), Recife - PE.
} 
Institución. La búsqueda del conocimiento sobre las medidas preventivas y como consecuencia sobre la mejor forma de cuidar al paciente, se convirtió en el interés de todos los profesionales que estaban directa e indirectamente vinculados al cuidado del paciente. En este escenario se capacitaron 2.152 profesionales en 215 grupos Consideraciones finales: Creyendo en la importancia del trabajo que realiza la educación permanente se percibe que es posible educar, de manera humanizada, que todos los profesionales son integrantes del proceso de aprendizaje, y que es fundamental para la comprensión de las necesidades y ansiedades vividas como parte de la construcción del nuevo sable en el ámbito laboral.

Palabras clave: Personal de salud, Infecciones por coronavirus, Educación continúe.

\section{INTRODUÇÃO}

A Educação Permanente (EP) baseia-se nas Portarias GM/MS no 198/2004: que institui a Política Nacional de Educação Permanente em Saúde (PNSPS) e a Portaria GM/MS no 1996/2007 que dispõe sobre as diretrizes para a implementação, como estratégia do Sistema Único de Saúde para a formação e o desenvolvimento de trabalhadores. É, portanto, um instrumento para mudanças e transformações dos serviços de saúde, integrando habilidades teóricas e práticas, se configurando como uma estratégia essencial para o aperfeiçoamento dos serviços de saúde, realizando ações integrativas, elaboração de políticas e também do controle social (CAMPOS KFC, et al., 2017; FRANÇA T, et al., 2017).

A EP também assume um papel de promover e inspirar experiências para que o profissional reconheça sua singularidade e se fortaleça, superando limites e dificuldades; e desenvolva também o seu potencial. A metodologia de trabalho é baseada em competências, que possui múltiplos significados, podendo ser entendida como uma capacidade de agir eficazmente em determinado tipo de situação, apoiada em conhecimentos, porém sem limitar-se a eles. A abordagem vai além do ensino com esquema e memorização, o próprio profissional é o ator envolvido no processo ensino-aprendizagem baseada nas necessidades de cada unidade de forma individualizada (FIGUEIREDO EBL, et al., 2017).

Em dezembro de 2019, surge o novo agente do coronavírus, através de casos de pneumonia registrados na cidade de Wuhan, China. Altamente patogênicos e responsáveis por causar síndrome respiratória aguda grave, denominado pela Organização Mundial da Saúde (OMS): Severe Acute Respiratory Syndrome Coronavirus (SARS-CoV), Middle East Respiratory Syndrome (MERS-CoV), também chamada de Corona Vírus Disease (COVID). Posteriormente passando a ser chamado de SARS-CoV-2 ou COVID-19 fazendo referência ao ano de surgimento (OMS, 2020; BRASIL, 2020a; BRASIL, 2021).

Pertencendo a uma família de vírus comuns em espécies de animais, pensou inicialmente que a disseminação acontecesse de animais a pessoas, mas o crescente número de pacientes não expostos a animais, indicou também a transmissão pessoa para pessoa. Rapidamente a doença espalhou-se pelo território chinês e, sequencialmente observou-se infecções pelo SARS-CoV-2 em outros países. Em janeiro de 2020, a OMS, declarou como uma Emergência de Saúde Pública de importância Internacional (ESPII), tornando-se uma pandemia (OMS, 2020; BRASIL, 2020a; BRASIL, 2020b).

Uma doença com período de incubação da infecção pelo coronavírus é de 5 dias, com intervalo que pode chegar até a 12 dias. Transmissibilidade em média de 7 dias após o início dos sintomas, porém dados preliminares sugerem que a transmissão possa ocorrer, mesmo sem o aparecimento de sinais e sintomas. Os sinais e sintomas clínicos mais referidos estão relacionados ao sistema respiratório, geralmente febre, tosse, dificuldade respiratória, mialgia, confusão, cefaléia, dor de garganta, rinorreia, dor no peito, náusea e vômito. Nos achados dos exames de imagem mais comuns temos pneumonia bilateral, manchas múltiplas e opacidade em vidro fosco (OMS, 2020; BRASIL, 2020c).

A Organização Pan-Americana de Saúde (OPAS) e o Ministério da Saúde recomendam como medidas básicas de prevenção, para evitar a proliferação do vírus: higiene das mãos. Cobrir a boca e o nariz com o antebraço quando tossir ou espirrar, fazendo uso de lenço descartável, evitar tocar olhos, nariz e boca, sem que as mãos estejam limpas, além do uso de máscara. Em Pernambuco o uso de máscaras se tornou obrigatório, no período de enfrentamento da emergência em saúde pública de importância internacional, de acordo com o Decreto do Governo do Estado de Pernambuco, no 48.969 (OMS, 2020; PERNAMBUCO, 2020a). 
Considerando que os pacientes suspeitos ou confirmados, levam os profissionais de saúde a manterem medidas de precaução padrão, de contato, gotículas e aerossóis, e para tanto faz-se necessário o uso de Equipamentos de Proteção Individual (EPI), indicados para assistência à saúde. Voltado a estas medidas temos como EPI: máscara cirúrgica, máscara de proteção respiratória N95/ PFF2 ou equivalente, luvas, óculos de proteção ou protetor de face (face shield), capote ou avental e gorro (BRASIL, 2020c)

Diante da pandemia foi necessário a estruturação de Protocolos Institucionais, baseados nos Protocolos do Ministério da Saúde. Uma das estratégias adotadas para disseminação das informações foram as ações educativas, sob a responsabilidade da Educação Permanente, que assumiu este grande desafio, apoiada pela gestão hospitalar, visando a sistematização dos cuidados com a segurança do paciente e dos profissionais (PERNAMBUCO, 2020b).

Nesse contexto de segurança do paciente e dos profissionais, os treinamentos abordavam os seguintes temas: definição de Covid-19, a situação epidemiológica da doença no mundo, no Brasil e em Pernambuco, principais sinais e sintomas, medidas de precaução padrão, gotícula, aerossóis e contato, importância do uso de EPI, demonstração das etapas de paramentação e desparamentação, e o passo a passo da higienização das mãos (PERNAMBUCO, 2020b).

Os profissionais que atuam dentro do ambiente hospitalar, seja diretamente ou indiretamente relacionados à assistência de saúde, estão propensos a contaminação pelo novo Covid-19. O Brasil é o segundo país em número de casos e de mortes de profissionais pela infecção, incluindo profissionais médicos, enfermeiros e técnicos de enfermagem (OMS, 2020; ABRASCO, 2020).

Diante da apreensão em capacitar a equipe multidisciplinar, foram implementadas ações de educação permanente sobre as medidas preventivas adotadas pela instituição, durante a pandemia do COVID-19, visando a prevenção dos profissionais. Neste trabalho relatamos a execução de treinamentos realizados sobre as medidas preventivas adotadas durante a pandemia do COVID-19, visando o fortalecimento e empoderamento por parte dos profissionais.

\section{RELATO DE EXPERIÊNCIA}

Com o início da Pandemia da COVID19, o estado de Pernambuco, através da Secretaria Estadual de Saúde de Pernambuco (SES/PE), solicitou ao Instituto de Medicina Integral Profo Fernando Figueira - IMIP, em março de 2020, que se tornasse referência para a assistência à gestante, puérpera e neonatos, como parte da estratégia de combate ao Coronavírus no estado. O desafio era enorme, a doença era desconhecida e as previsões ameaçadoras, ainda assim, o IMIP pelo seu histórico, assumiu o compromisso de assistir à população nesse cenário crítico.

Diante do aumento de casos de pacientes com a Síndrome Respiratória Aguda Grave (SRAG) e da infecção humana pelo novo coronavírus (COVID-19), visando atender a esta demanda, o IMIP, em parceria com a SES/PE pactuou a abertura de novos leitos na instituição, sendo 50 leitos de Unidade de Terapia Intensiva (UTI) adulto, 53 leitos de enfermaria gestante COVID e 20 leitos de UTI Neonatal COVID. No âmbito da Educação Permanente, foi um desafio para a gestão proporcionar a qualificação dos profissionais, considerando esta ação como item imprescindível para uma melhor qualidade do serviço prestado.

A capacitação profissional em serviço da Instituição aconteceu de forma permanente e tais atividades continuam sendo desenvolvidas. Como estratégias de gestão foi articulado treinamentos de forma sistemática, executado pelos setores de Educação Permanente, Comissão de Controle de Infecção Hospitalar $(\mathrm{CCIH})$ e Gerência de Risco, visando primordialmente à segurança dos profissionais, abordando os seguintes temas: COVID-19, a situação epidemiológica no Brasil e em Pernambuco, sinais e sintomas, medidas de precaução padrão, uso de EPI, paramentação e desparamentação, e execução pelos participantes do passo a passo da higienização das mãos.

Perante a pandemia que estamos vivenciando, a gestão compartilhada dos diversos serviços propostos pelo hospital, precisou assumir um papel ainda mais proativo, na busca da qualidade do serviço prestado, além da garantia da segurança dos profissionais e dos pacientes. Para execução dos treinamentos, pudemos 
vivenciar três momentos distintos: a elaboração dos treinamentos de acordo com os protocolos do Ministério da Saúde e Institucionais, a execução e a observação da prática com sua atualização permanente de acordo com as necessidades grupais.

Durante a elaboração dos treinamentos de acordo com os protocolos do Ministério da Saúde e Institucionais, vivenciamos várias etapas em ter que refazer os pensamentos e a melhor forma de conduzir as orientações sobre o COVID-19 e suas medidas de precaução, pois estávamos diante de uma doença ainda desconhecida que precisava ser estudada e a cada nova descoberta, havia necessidade de atualizações dos protocolos ministeriais e consequentemente institucional.

$\mathrm{Na}$ execução dos treinamentos diante de uma pandemia houve um fortalecimento da Educação Permanente dentro da Instituição, tornando-se mais visível para todas as áreas e não apenas para a área de enfermagem, havendo uma procura de outras áreas pela capacitação, fato este que antes acontecia de forma pontual. Além disso, a EP culturalmente, trazia uma imagem de ser solicitada apenas quando era identificado uma necessidade particular de determinadas áreas, e era realizada no intuito de correção para a melhoria da prática assistencial. A busca pelo conhecimento sobre as medidas de prevenção e a melhor forma de cuidar do paciente, passou a ser interesse de todos os profissionais que estavam direta e indiretamente ligados ao cuidado com o paciente.

No período de fevereiro a agosto tivemos aproximadamente 215 grupos de treinamentos, respeitando o limite de 10 pessoas no máximo em cada grupo e o distanciamento social; os treinamentos aconteceram sempre em locais amplos (auditórios, salas de atendimentos, e em alguns setores), ventilados e todos os profissionais em uso de máscara, num total de 2.152 treinados. Sendo: 12,1\% (262) em fevereiro, $26,9 \%$ (577) em março, 20,9\% (448) em abril, 8,4\% (182) em maio, 15,6\% (334) em junho, 12,1\% (262) em julho e $4,0 \%$ (87) em agosto.

Quanto à categoria profissional, das pessoas capacitadas, foram 21,6\% (465) profissionais de nível superior (médicos, enfermeiros, fisioterapeutas, assistentes sociais, nutricionistas, dentistas e farmacêuticos), 69,0\% (1.485) nível médio (Técnicos de enfermagem, administrativos, porteiros, técnico de laboratório, auxiliar de nutrição, auxiliar de farmácia, auxiliar de saúde bucal e acadêmicos), 3,7\% (80) nível elementar (auxiliar de higienização, manutenção e maqueiros) e 5,7\% (122) não identificados.

A necessidade de acompanhar as atualizações, a aspiração em acertar nos cuidados individuais quanto às medidas de precaução, foi perceptível diante de um público treinado que questionava, discutia as dúvidas, buscava sempre mais conhecimentos, se co-responsabilizavam com os temas abordados, trazendo à tona tudo que surgia de novo e que buscava executar as práticas de forma correta, item este observado na atenção prestada as capacitações, fez emergir juntamente um sentimento de fortalecimento em conjecturar que é possível acreditar na relevância de uma Educação Permanente influente e consistente para a prática assistencial.

Com a observação da prática, e sua atualização permanente de acordo com as necessidades grupais, estamos em constante progressão e acompanhamento, para que os profissionais consigam desempenhar suas atividades laborais buscando o cuidado pessoal prestando ao paciente um cuidado humanizado e seguro.

Durante a pandemia, foi possível perceber que a Educação Permanente em seu papel de promover e inspirar a troca de experiências desempenhou um papel fundamental e singular, proporcionando aos profissionais o reconhecimento de suas limitações, o fortalecimento de seus conhecimentos e a possibilidade de juntos superar limites e dificuldades através do desenvolvimento do seu potencial.

\section{DISCUSSÃO}

A enfermagem sempre esteve atuante na EP, política instituída desde 2004 como estratégia do SUS para a formação e desenvolvimento dos trabalhadores. A EP da Instituição foi criada a fim de atender as demandas de treinamento da equipe de enfermagem e demais categorias profissionais visando à promoção do desenvolvimento de atividades de ensino aprendizagem (BRASIL, 2004; BAPTISTA GL, 2009). 
No organograma da instituição, a EP está diretamente ligada a Diretoria de Enfermagem e por ser um hospital escola de grande porte, a E, sempre assumiu as capacitações voltadas mais diretamente aos profissionais de Enfermagem, trabalhando temas solicitados e planejados junto com a Diretoria visando uma prática assistencial segura ao profissional e paciente. Onde o próprio profissional é o ator envolvido no processo ensino-aprendizagem baseado nas necessidades de cada unidade de forma individualizada (BRASIL, 2007; BAPTISTA GL, 2009).

Com o cenário epidemiológico atual a EP da instituição engloba uma nova abrangência, capacitar todos os trabalhadores da saúde, onde o conhecimento precisou ser adaptado aos diversos saberes como no estudo Silva CT, et al. (2016), onde foi relatada a necessidade de adotarem estratégias diversas para atingir a aprendizagem de usuários, estudantes de saúde e docentes.

No âmbito da Educação Permanente, o desafio para a gestão foi proporcionar a qualificação dos profissionais, considerando esta ação como item imprescindível para uma melhor qualidade do serviço prestado. A capacitação profissional em serviço da Instituição aconteceu de forma permanente e tais atividades continuam sendo desenvolvidas. Como estratégias de gestão os treinamentos acontecem de forma sistemática, visando primordialmente à segurança dos profissionais e dos pacientes (CAVALCANTI FOL, et al., 2018; DUARTE PLF, et al., 2018).

Assim como no estudo de Silva LAA, et al. (2016), além da capacitação dos profissionais da instituição sobre os protocolos de prevenção da disseminação do Covid-19, outro resultado importante desse programa de treinamentos foi o reconhecimento da EP por toda instituição, como um serviço transformador das práticas assistenciais, através de processos dinâmicos de ensino aprendizagem e reflexões da prática. $O$ estudo de Silva KL, et al. (2017), também retrata a EP com grande potencialidade para transformação da prática dos profissionais de saúde.

Durante a prática da EP foi observado ainda, a capacidade de adaptação, tendo em visa as diversas alterações à medida que se iam tendo novas informações sobre a transmissão do Covid-19. A cada atualização dos protocolos de prevenção das autoridades competentes, a capacitação era reformulada, conjuntamente aos protocolos institucionais e os materiais de divulgação. Essa capacidade também foi observada no estudo de Feliciano $A B$, et al. (2020), outras capacidades descritas nesse estudo foram "heroísmo versus humanidade" e "coordenação de cuidados e relações de poder", que também foram observados em nossa experiência.

Para os profissionais o papel desempenhado pela EP, pode promover através do ensino-aprendizagem, estratégias para a oferta de uma prática assistencial firmada em processos institucionais, de forma segura ao paciente. Foi perceptível as contribuições dos treinamentos para a melhoria da assistência e dos processos de trabalho, a promoção de capacitações através de conhecimentos técnicos e científicos gerou a confiança no melhor desempenho das atividades diárias. A ampliação dos conhecimentos promove desenvolvimento e parceria nas equipes, além de notoriamente o cuidado com a saúde de forma individual e coletiva (LAMANTE MPS, et al., 2019; MELLO AL, et al., 2018; PEREIRA LDÁ, et al., 2018; SOUSA MST, et al., 2015).

A pandemia trouxe aos profissionais um turbilhão de sentimentos, alguns percebidos e verbalizados durante os treinamentos, como o medo de adoecer, da morte, da transmissão para as pessoas próximas. Mas também foram compartilhados sentimentos positivos, como a empatia, o senso de coletividade, o apoio e cuidado mútuo, percebidos através das falas e também do empenho individual no seguimento dos protocolos e medidas preventivas.

\section{CONSIDERAÇÕES FINAIS}

A atuação da Educação Permanente durante uma pandemia foi desafiador; acompanhar as inúmeras atualizações de protocolos e junto a isso a responsabilização pela capacitação das equipes multiprofissionais, fez emergir a notoriedade da EP. Apresentando a sua potencialidade na propagação e trocas contínuas de conhecimento entre os profissionais das diversas áreas, evidenciou ainda que todos os profissionais são atores importantes no processo de aprendizagem, e que é essencial a compreensão das necessidades e angústias vivenciadas como parte da construção do novo saber dentro do ambiente de trabalho. 


\section{REFERÊNCIAS}

1. ABRASCO. Em defesa da vida: solidariedade aos profissionais de saúde mortos por Covid-19. Disponível em:https://www.abrasco.org.br/site/noticias/posicionamentos-oficiais-abrasco/em-defesa-da-vida-solidariedade-aosprofissionais-de-saude-mortos-por-covid-19/49520/. Acesso em: 28 de dezembro de 2020.

2. BAPTISTA GL, CUNHA MI. A dimensão educativa nos cursos de graduação em enfermagem: um estudo sobre a formação inicial. Revista Conhecimento Online 2009; 1 (1): 1-17.

3. BRASIL. Portaria no 198/2004 GM/MS. Institui a Política Nacional de Educação Permanente em Saúde como estratégia do Sistema Único de Saúde para a formação e o desenvolvimento de trabalhadores para o setor e dá outras providências. $2004 . \quad$ Disponível: http://bvsms.saude.gov.br/bvs/saudelegis/gm/2017/MatrizesConsolidacao/comum/13150.html. Acessado em: 04 de janeiro de 2021.

4. BRASIL. Portaria no 1996/2007 GM/MS. Dispõe sobre as diretrizes para a implementação da Política Nacional de Educação Permanente em $2007 . \quad$ Saúde. http://bvsms.saude.gov.br/bvs/saudelegis/gm/2007/prt1996_20_08_2007.html. Acessado em: 22 de dezembro de 2020.

5. BRASIL. Diretrizes para diagnóstico e tratamento da COVID-19. 2020a. Disponível em: https://portalarquivos.saude.gov.br/images/pdf/2020/April/18/Diretrizes-Covid19.pdf. Acessado em: 20 de dezembro de 2020.

6. BRASIL. Protocolo de manejo clínico para o novo coronavírus (2019-ncov). 2020b. Disponível em: https://12ad4c9289c7-4218-9e11-0ee136fa4b92.filesusr.com/ugd/3293a8_66b488ab08224c8da25c038dfa972c43.pdf. Acessado em:20 de dezembro de 2020.

7. BRASIL. Nota Técnica no 04/2020 GVIMS/GGTES/ANVISA. Orientações para serviços de saúde: medidas de prevenção e controle que devem ser adotadas durante a assistência aos casos suspeitos ou confirmados de infecção pelo novo coronavírus (sars-cov-2). 2020c. Disponível em: file:///C:/Users/gabrielamsr/Downloads/NOTA_TECNICA_GVIMS_GGTES_ANVISA_04_2020_Reviso_27.10.2020.p df. Acesso em: 22 dezembro de 2020.

8. BRASIL. Boletim Epidemiológico - Secretaria de Vigilância em Saúde: Infecção humana pelo novo coronavírus (2019ncov) $\quad n^{\circ} \quad 44.2021$ Disponível 2 em: https://www.gov.br/saude/ptbr/media/pdf/2021/janeiro/07/boletim_epidemiologico_covid_44.pdf. Acesso em: 04 de janeiro de 2021.

9. CAMPOS KFC, et al. Educação Permanente nos serviços de saúde. Esc Anna Nery 2017; 21(4): e20160317.

10. CAVALCANTI FOL, GUIZARDI FL. Educação Continuada ou Permanente em Saúde? Análise da produção PanAmericana da saúde. Trab. educ. saúde, 2018; 16(1): 99-122.

11. DUARTE PLF, KAEFER CO.As potencialidades da educação permanente em saúde: uma inquietação necessária para o fazer profissional. Disciplinarum Scientia. Série: Ciências da Saúde, Santa Maria, 2018; 19(3): 449-465.

12. FELICIANO AB, et al. A pandemia de covid-19 e a educação permanente em saúde. Cadernos da Pedagogia, 2020; 14(29) 120-135.

13. FIGUEIREDO EBL, et al. Dez anos da Educação Permanente como política de formação em saúde no Brasil: um estudo das teses e dissertações. Trab. educ. saúde, 2017; 15(1): 147-162.

14. FRANÇA T, et al. Política de Educação Permanente em Saúde no Brasil: a contribuição das Comissões Permanentes de Integração Ensino-Serviço. Ciência \& Saúde Coletiva, 2017; 22(6): 1817-1828.

15. LAMANTE MPS, et al. A educação permanente e as práticas em saúde: concepções de uma equipe multiprofissional. Revista Pesquisa Qualitativa, 2019; 7(14):230-244.

16. MELLO AL, et al. Estratégia organizacional para o desenvolvimento de competências de enfermeiros: possibilidades de Educação Permanente em Saúde. Esc. Anna Nery, 2018; 22(1): e20170192.

17. OMS. Declaração emergência de saúde pública internacional em relação a novo coronavírus. Disponível em: https://www.paho.org/bra/index.php?option=com_content\&view=article\&id=6100:oms-declara-emergencia-de-saudepublica-de-importancia-internacional-em-relacao-a-novo-coronavirus\&ltemid=812. Acesso em: 08 de dezembro de 2020.

18. PEREIRA LDÁ, et al. Educação Permanente em saúde: uma prática possível. Rev enferm UFPE on line, 2018; 12(5): 1469-79.

19. PERNAMBUCO. Decreto no 48.696/2020. Disponível em: https://www.amupe.org/wpcontent/uploads/2020/03/Governo-de-Pernambuco-DECRETO-N\%C2\%BA-48.969-DE-23-DE-ABRIL-DE-2020-.pdf. Acesso em: 22 de dezembro de 2020.

20. PERNAMBUCO. Secretaria de Saúde - Segurança do Paciente: recomendações aos Núcleos de Segurança do Paciente no enfrentamento da Covid-19. 2020. Disponível em: http://portal.saude.pe.gov.br/sites/portal.saude.pe.gov.br/files/manual_seguranca_do_paciente_agosto2020.pdf. Acesso em: 20 de dezembro de 2020.

21. PERNAMBUCO. Secretaria de Saúde - Boletim Covid-19 no 310. 2021. Disponível em: https://drive.google.com/file/d/1MF4SIO004v9PF6L_gixKDQaFcvU0lp6F/view. Acesso em: 04 de janeiro de 2021.

22. SILVA CT, et al. Residência multiprofissional como espaço intercessor para a educação permanente em saúde. Texto contexto - enferm., 2016; 25(1), e2760014.

23. SILVA LAA, et al. Avaliação da educação permanente no processo de trabalho em saúde. Trab. educ. Saúde, 2016; 14(3), 765-781.

24. SILVA KL, et al. A construção da educação permanente no processo de trabalho em saúde no estado de Minas Gerais, Brasil. Esc. Anna Nery, 2017; 21(4), e20170060.

25. SOUSA MST, et al. A percepção dos enfermeiros sobre educação permanente em saúde no contexto da estratégia saúde da família de Sobral (CE). Revista Interfaces: Saúde, Humanas e Tecnologia, 2015; 3(1). 